\title{
Tecnologías de la Información y la Comunicación y diversidad funcional. Conocimiento y formación del profesorado en Navarra
}

\section{Information and Communication Technologies and functional diversity: knowledge and training of teachers in Navarra}

Eloy López Meneses. Universidad Pablo de Olavide. Grupo Eduinnovagogía® (HUM-971). elopmen@upo.es

José Fernández Cerero. Universidad de Sevilla. jfercerero@gmail.com

\section{RESUMEN.}

En los últimos años se ha avanzado bastante al analizar las TIC como instrumentos significativos que favorecen la inclusión de las personas con diferentes tipos de diversidad cognitivas, sensoriales, o motóricas. A pesar de que existen prácticas de calidad vinculadas al uso de las TIC y cada vez un mayor interés en el uso educativo de estas herramientas tecnológicas, sigue existiendo una gran preocupación por resolver las diferentes dificultades en competencia digital que presentan los docentes, y más aún en contextos de diversidad. Por tanto, el profesorado se configura como un elemento clave para lograr una plena inclusión de las TIC en las aulas. En este marco, se pretende realizar un diagnóstico en relación con la formación y conocimiento del profesorado de Educación Primaria, en activo, en relación con las TIC aplicadas a personas con diversidad funcional. En el aspecto metodológico, se ha procedido a la realización de un diseño de investigación de corte cualitativo, desarrollado desde el enfoque de la Grounded Theory. La información se ha obtenido a partir del análisis de 52 entrevistas realizadas a profesionales del sector educativo del estado español (miembros de equipos directivos, coordinadores TIC, directores y asesores tecnológicos de centros de formación). Entre las conclusiones podemos destacar la baja capacitación del profesorado, el escaso desarrollo de actividades de formación y que las principales barreras que obstaculizan dicha realización de actividades de formación en TIC y diversidad funcional en la Comunidad de Navarra vienen determinadas en primer lugar por factores económicos y la falta de tiempo.

\section{PALABRAS CLAVE.}

Tecnologías de la Información y Comunicación, diversidad funcional, discapacidad, formación del profesorado, tecnología educativa, accesibilidad.

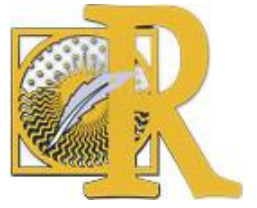

Fecha de recepción: 02-11-2019 Fecha de aceptación: 09-12-2019 


\section{ABSTRACT.}

In recent years, considerable progress has been made in analyzing ICTs as significant instruments that favor the inclusion of people with different types of cognitive, sensory, or motor diversity. Although there are quality practices linked to the use of ICT, and an increasing interest in the educational use of these technological tools, there is still a great concern as how to solve the different difficulties in digital competence that teachers present, and even more in contexts of diversity. Therefore, teachers are configured as a key element to achieve full inclusion of ICT in the classroom. In this framework, it is intended to make a diagnosis in relation to the training and knowledge of active Primary Education teachers, in relation to ICT applied to people with functional diversity. Under the methodological aspect, a qualitative research design has been carried out, developed from the Grounded Theory approach. The information has been obtained from the analysis of 52 interviews with professionals in the education sector of the Spanish state of Navarra (members of management teams, ICT coordinators, directors and technological advisors of training centers). Among the conclusions we place a stress on the low training of teachers, the low development of training activities, and also note that the main barriers that hinder such completion of ICT training activities and functional diversity in the Community of Navarra are determined primarily by economic factors and the lack of time.

\section{KEY WORDS.}

Information and Communication Technologies, functional diversity, disability, teacher training, educational technology, accessibility.

\section{Introducción.}

El presente estudio forma parte de un proyecto más amplio de investigación titulado "Diagnóstico y formación del profesorado para la incorporación de las TIC en alumnado con diversidad funcional" financiado en el marco del Plan Estatal de Fomento de la Investigación Científica y Técnica de Excelencia 2013-2016 (DIFOTICYD EDU2016 75232-P).

La implementación de las TIC en el sistema educativo actual está generando un perfil docente muy diferente al de hace años. Este hecho abre nuevas vías de investigación en educación centradas en la formación permanente del profesorado, es decir, en la concepción y el grado de formación y dominio que el docente tenga sobre estos recursos.

Dicha formación debe centrarse en dotar al profesorado de los conocimientos, habilidades y competencias necesarias para el uso de las TIC como un recurso para acercar los procesos de enseñanza-aprendizaje a los estudiantes y más aún, a aquellos con necesidades educativas especiales. Esto permitirá el desarrollo de nuevos contextos educativos que tengan en cuenta las diferencias individuales del alumnado (Shin, 2015).

En esta línea, este trabajo incide en el ámbito de la formación continua del profesorado sobre el uso de las TIC para personas con diversidad funcional. Además, consideramos que este estudio es relevante ya que, en primer lugar, son muy escasos los estudios sobre la formación en TIC especialmente para personas con diversidad funcional y, en segundo lugar, ofrece la opinión directa del profesorado, su nivel de formación y los principales obstáculos que dificultan el desarrollo de actividades formativas. Todo ello permitirá reparar en los factores

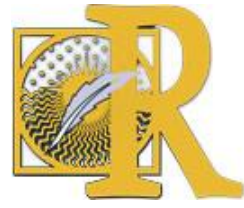


que inciden en las prácticas pedagógicas con TIC destinadas al alumnado con diversidad funcional.

Teniendo en cuenta este contexto, en el panorama del contexto español el volumen de producción de trabajos relacionados con la problemática de la capacitación en TIC de los profesores y las competencias que necesitan para su utilización es abundante (Fernández Batanero \& Bermejo, 2012; Rosario \& Vázquez, 2012; Rangel \& Peñalosa, 2013 y Ortíz, Almazán, Peñaherrera \& Cachón, 2014) y concluyen con que los docentes no se sienten suficientemente preparados para incluir la tecnología de forma eficaz en su práctica diaria, debido al uso instrumental que se hace de la misma (Roig, Ferrández, Rodríguez-Cano \& Crespo, 2012; Álvarez \& Gisbert, 2015,Tello \& Cascales, 2015 y Cabero, FernándezBatanero \& Barroso, 2016).

La utilización inapropiada que el profesorado hace de las TIC en el aula aumenta si, además, tenemos en cuenta las barreras que obstaculizan la formación del profesorado. Diversas son las investigaciones que ponen de manifiesto los factores que impiden la implantación de las TIC en los centros escolares. Entre ellas, destacamos aquellas que hacen referencia a barreras extrínsecas (falta de tiempo, recursos y financiación para realizar la formación) y, las barreras intrínsecas, (atribuidas a la actitud del profesorado) (Barrantes, Casas \& Luengo, 2011; Ramírez, Cañedo \& Clemente, 2011; González \& De Pablos, 2015 y Villalba, GonzálezRivera\& Díaz-Pulido, 2017).

Supuesto esto, si hablamos de las competencias TIC necesarias para utilizarlas con personas con diversidad funcional, podemos comprobar el poco volumen de trabajos existentes a nivel internacional (Altinay y Altinay, 2015; Turner-Cmuchal \& Aitken, 2016; Alper y Goggin, 2017, Hollier, 2017, Fernández Batanero, 2020). En el contexto nacional los escasos estudios que tratan la capacitación TIC del profesorado destacan también la falta de formación y conocimiento que tienen los docentes respecto a los diferentes tipos de tecnologías que pueden utilizarse con el alumnado, las posibilidades que ofrecen, y las funciones para las que pueden ser utilizadas (Roig, Ferrández, Rodríguez-Cano \& Crespo, 2012; Tello \& Cascales, 2015; Morales \& Llorente Cejudo, 2016 y Fernández-Batanero \& Rodríguez, 2017, Fernández Batanero, Cabero \& López, 2019).

La escasa existencia de trabajos sobre esta temática, repercute negativamente en la utilización de las TIC, impidiendo hacer más accesible la información y potenciar las capacidades de las personas, en este caso con diversidad funcional, ayudando a que sus dificultades se vieran paliadas y/o se redujeran al mínimo (Homero, Tejedor \& Calvo, 2017).

\section{Material y métodos.}

Propósito y pregunta de investigación.

Partiendo del contexto anterior, la finalidad de la investigación desarrollada ha sido conocer la percepción de directores, jefes de estudios y coordinadores TIC de centros educativos, así como directores y asesores tecnológicos de centros de formación, sobre el nivel de formación y conocimiento tecnológico que posee el profesorado de Educación Primaria de la Comunidad Autónoma de Navarra respecto a la aplicación de las TIC para alumnado con diversidad funcional. Para abordar este objetivo, se llevó a cabo un estudio exploratorio

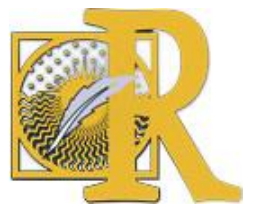

Fecha de recepción: 02-11-2019 Fecha de aceptación: 09-12-2019 
desarrollado en el segundo trimestre de 2017. Se exploran las siguientes preguntas de investigación:

RQ1 ¿Se encuentra el profesorado de Educación Primaria de la Comunidad de Navarra, suficientemente preparado y concienciado para el uso y aplicación de las TIC en alumnado con diversidad funcional?

RQ2 ¿Condiciona el tipo de centro docente el nivel de competencia del profesorado para usar las TIC en alumnado con diversidad funcional?

RQ3 ¿Qué factores se asocian a la promoción y el desarrollo de experiencias de formación sobre usos de las TIC en alumnado con diversidad funcional?

Teniendo en cuenta los objetivos planteados, se aplica una metodología cualitativa, sustentada desde una perspectiva paradigmática interpretativa, y desde una ontología y epistemología claramente naturalistas. Específicamente, nuestra aproximación interpretativa se basa en el método de la Teoría Fundamentada o Grounded Theory, propuesto por Glaser y Strauss. Este método nos permite robustecer el proceso de transformación de los datos desde la conceptualización teórica y la generación de teoría formal fundamentada en el área de la educación de personas con diversidad funcional mediante TIC.

\section{Participantes.}

La muestra del estudio la conforman52 profesionales del sector educativo. La selección de elementos se ha hecho por unidades grupales naturales, en nuestro caso, el tipo de centro. Por lo tanto, el objeto del estudio han sido centros educativos de titularidad pública, privada y concertada, así como centros de formación del profesorado (CEP en adelante), ubicados en la Comunidad de Navarra.

Tabla 1. Distribución Comunidad Autónoma y tipo de centro

\begin{tabular}{lcccc}
\hline & CEP & CEIP & CPC & CP \\
\hline Navarra & $15 \%$ & $54 \%$ & $23 \%$ & $18 \%$
\end{tabular}

Nota: CEP (Centro de Formación del Profesorado); CEIP (Centro Educativo de Educación Infantil y Primaria); CPC (Centro Público Concertado); CP (Centro Privado).

La distribución de las entrevistas realizadas ha sido la siguiente: del total de la muestra el $31 \%$ se corresponde a directores de centros educativos, el $15 \%$ hace referencia a jefes de estudios, el $38 \%$ a coordinadores $\mathrm{TIC}$, el $8 \%$ se corresponde a directores de centros de formación del profesorado y el $8 \%$ a asesores tecnológicos de centros de formación del profesorado. Por último, señalamos que el $62 \%$ del entrevistado son hombres y el $38 \%$ son mujeres.

Las categorías seleccionadas para el estudio han girado en torno a: aspectos sociodemográficos (tipo de centro, cargo que ocupa, sexo y antigüedad en el cargo); concepto de diversidad funcional; concepto de discapacidad; beneficios TIC para personas con diversidad funcional; concienciación y preparación del profesorado; desarrollo de

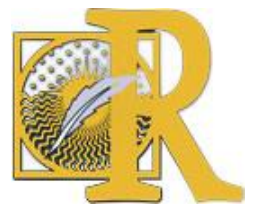

Fecha de recepción: 02-11-2019 Fecha de aceptación: 09-12-2019 
experiencias de formación; promoción de la formación; barreras para el desarrollo de planes de formación; prioridad en la formación.

Tabla 2. Sistema categorial

\begin{tabular}{|c|c|c|}
\hline Categorías y definición & Subcategorías & Evidencias \\
\hline $\begin{array}{l}\text { Aspectos } \\
\text { sociodemográficos: sitúan } \\
\text { la información aportada por } \\
\text { los entrevistados en un } \\
\text { determinado } \\
\text { contexto/entorno. }\end{array}$ & $\begin{array}{l}\text { - Tipo de centro educativo. } \\
\text { - Cargo que ocupa. } \\
\text { - Sexo. } \\
\text { - Antigüedad en el cargo. }\end{array}$ & $\begin{array}{l}\text { - Público (CEIP); Privado (CP); Privado Concertado } \\
\text { (CPC); Centro de formación del profesorado (CEP) } \\
\text { - Director/ Director CEP; Jefe de estudios; Coordinador } \\
\text { TIC/Asesor TIC CEP. } \\
\text { - Hombre; Mujer. } \\
\text { - } 0 \text { a } 2 \text { años; } 3 \text { a } 5 \text { años; } 6 \text { a } 10 \text { años; } 11 \text { o más. }\end{array}$ \\
\hline \multirow{2}{*}{$\begin{array}{l}\text { Concepto de diversidad } \\
\text { funcional: diferencia de } \\
\text { funcionamiento de una } \\
\text { persona al realizar las tareas } \\
\text { habituales (desplazarse, leer, } \\
\text { agarrar, comunicarse, } \\
\text { relacionarse, etc.) de manera } \\
\text { diferente a la mayoría de la } \\
\text { población (Romañach y } \\
\text { Lobato, 2005). }\end{array}$} & \multirow[t]{2}{*}{$\begin{array}{l}\text { Conocimiento o no del término } \\
\text { diversidad funcional por parte } \\
\text { de los entrevistados. }\end{array}$} & $\begin{array}{l}\text { Respuesta correcta: "Entiendo que nos referimos a las } \\
\text { distintas formas en que las personas afrontamos las } \\
\text { diferentes tareas o actividades en la vida diaria, en } \\
\text { función de las capacidades de cada persona" } \\
\text { (ENTRE.12). }\end{array}$ \\
\hline & & $\begin{array}{l}\text { Respuesta incorrecta: "Personas que no pueden hacer } \\
\text { algo". (ENTRE.31). }\end{array}$ \\
\hline \multirow[t]{2}{*}{$\begin{array}{l}\text { Concepto de discapacidad: } \\
\text { término general que abarca } \\
\text { las deficiencias, las } \\
\text { limitaciones de la actividad y } \\
\text { las restricciones de la } \\
\text { participación (Organización } \\
\text { Mundial de la Salud, 2001). }\end{array}$} & \multirow[t]{2}{*}{$\begin{array}{l}\text { Conocimiento o no del término } \\
\text { discapacidad por parte de los } \\
\text { informantes claves. }\end{array}$} & $\begin{array}{l}\text { Respuesta correcta: "Siempre creo que hemos asociado } \\
\text { discapacidad a tener una limitación concreta para hacer } \\
\text { algo. Prefiero el término diversidad funcional. Por } \\
\text { ejemplo: una persona invidente en lugar de enfocar que } \\
\text { tiene una discapacidad, por ejemplo, para leer un } \\
\text { libro, pienso que hay que enfocarlo mejor como que } \\
\text { tiene una forma diferente a la mía de acceder a esa } \\
\text { información. Esta persona puede leer con otro código } \\
\text { diferente al mío, pero no está discapacitada para } \\
\text { hacerlo, sino que lo hará de una forma diferente." } \\
\text { (ENTRE.08). }\end{array}$ \\
\hline & & $\begin{array}{l}\text { Respuesta incorrecta: "Los alumnos que tienen } \\
\text { problemas para ciertas cosas; van un poco más lentos } \\
\text { que los demás.". (ENTRE.22). }\end{array}$ \\
\hline \multirow{4}{*}{$\begin{array}{l}\text { Beneficios TIC para } \\
\text { personas con diversidad } \\
\text { funcional: mejora que } \\
\text { experimenta una persona } \\
\text { gracias a algo que se le hace } \\
\text { o se le da. }\end{array}$} & Accesibilidad. & $\begin{array}{l}\text { "Los beneficios que aportan las TIC creo que son muy } \\
\text { importantes, porque permiten acceder a una gran } \\
\text { cantidad y variedad de recursos que posibilitan un mayor } \\
\text { aprovechamiento de las capacidades de las personas". } \\
\text { (ENTRE.48). }\end{array}$ \\
\hline & Comunicación y expresión. & $\begin{array}{l}\text { "Hay niños que a lo mejor no se pueden comunicar } \\
\text { oralmente y a través de las TIC podrían". (ENTRE.29). }\end{array}$ \\
\hline & Facilita el aprendizaje. & $\begin{array}{l}\text { "Pueden ayudar también a compensar en cierta medida" } \\
\text { algunas limitaciones que estas personas puedan tener". } \\
\text { (ENTRE.37). }\end{array}$ \\
\hline & Motivación. & $\begin{array}{l}\text { "Yo creo que es muy motivador para los niños porque } \\
\text { les ayudan, son más visuales. Tenemos un alumno con } \\
\text { adaptaciones curriculares y con él usamos una Tablet de } \\
\text { forma individual.". (ENTRE.30). }\end{array}$ \\
\hline \multirow{2}{*}{$\begin{array}{l}\text { Concienciación } \quad \text { y } \\
\text { preparación } \\
\text { profesorado: } \\
\text { Concienciación: percepción } \\
\text { de los entrevistados acerca } \\
\text { de si los profesores de } \\
\end{array}$} & Concienciado y preparado. & $\begin{array}{l}\text { "Sí, yo pienso que sí. Además, hoy en día el profesorado } \\
\text { que tiene algún tipo de dudas siempre puede consultar } \\
\text { al responsable TIC del centro." (ENTRE.16). }\end{array}$ \\
\hline & Concienciado sí, preparado no. & $\begin{array}{l}\text { "Concienciado a nivel moral o actitudinalmente pienso } \\
\text { que sí. Sin embargo, en la práctica creo que podemos } \\
\text { seguir mejorando. En cuanto a la preparación creo que }\end{array}$ \\
\hline
\end{tabular}

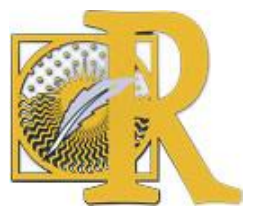

Fecha de recepción: 02-11-2019 Fecha de aceptación: 09-12-2019

López-Meneses, E. \& Fernández-Cerero, J. F. (2020). Tecnologías de la Información y la Comunicación y

diversidad funcional. Conocimiento y formación del profesorado en Navarra.

International Journal of Educational Research and Innovation (IJERI), 14, 59-75

ISSN: 2386-4303 DOI https://doi.org/10.46661/ijeri.4407 


\begin{tabular}{|c|c|c|}
\hline \multirow{2}{*}{$\begin{array}{l}\text { primaria son conscientes de } \\
\text { la importancia de realizar } \\
\text { actividades de formación. } \\
\text { Preparación: Nivel de } \\
\text { formación del profesorado. }\end{array}$} & & $\begin{array}{l}\text { queda aún un largo camino por recorrer. Creo que el } \\
\text { margen de mejora es muy amplio" (ENTRE.05). }\end{array}$ \\
\hline & Ni concienciado, ni preparado. & $\begin{array}{l}\text { "El profesorado de educación primaria no estamos } \\
\text { preparados. En todo caso lo que hacemos es pedir } \\
\text { asesoramiento a los especialistas de P.T. y A.L. del } \\
\text { centro.". (ENTRE.42). }\end{array}$ \\
\hline \multirow{2}{*}{$\begin{array}{l}\text { Desarrollo de experiencias } \\
\text { de formación: acciones } \\
\text { formativas elaboradas y } \\
\text { desarrolladas en los centros } \\
\text { educativos de Educación } \\
\text { Primaria. }\end{array}$} & \multirow{2}{*}{$\begin{array}{l}\text { Desarrollo o no de } \\
\text { experiencias de formación en } \\
\text { los centros de Educación } \\
\text { Primaria. }\end{array}$} & $\begin{array}{l}\text { "Sí, sí que se desarrollan. Recientemente hemos } \\
\text { recibido unos cursos de formación de Tablets". } \\
\text { (ENTRE.50). }\end{array}$ \\
\hline & & $\begin{array}{l}\text { "No, sinceramente desconozco que se lleven a cabo } \\
\text { este tipo de experiencias.". (ENTRE.32). }\end{array}$ \\
\hline \multirow{2}{*}{$\begin{array}{l}\text { Promoción de la } \\
\text { formación: favorecer de } \\
\text { algún modo que una acción } \\
\text { se desarrolle o que aumente } \\
\text { un aspecto positivo hacia } \\
\text { ella. }\end{array}$} & \multirow[t]{2}{*}{$\begin{array}{l}\text { Promoción o no de iniciativas } \\
\text { de formación } \\
\text { (Cursos, grupos de trabajo, } \\
\text { proyectos o planes de trabajo, } \\
\text { reuniones, seminarios) }\end{array}$} & $\begin{array}{l}\text { "Sí se promueven. Desde el CEP con una oferta } \\
\text { bastante amplia de ofertas de } \\
\text { formación, una oferta que es más amplia desde hace un } \\
\text { tiempo con la posibilidad } \\
\text { que nos ofrece la Teleformación" (ENTRE.26). }\end{array}$ \\
\hline & & $\begin{array}{l}\text { "Ni se promueve ni se incentiva. A día de hoy } \\
\text { desconozco que existan programas tan específicos" } \\
\text { (ENTRE.40). }\end{array}$ \\
\hline \multirow{5}{*}{$\begin{array}{l}\text { Barreras para el desarrollo } \\
\text { de planes de formación: } \\
\text { obstáculo que impide o } \\
\text { dificulta la realización de } \\
\text { actividades de formación. }\end{array}$} & Actitud del profesorado. & $\begin{array}{l}\text { "Más que barreras creo que a veces es necesario un } \\
\text { poco más de iniciativa.". (ENTRE.04). }\end{array}$ \\
\hline & Económicas. & $\begin{array}{l}\text { "Primero, la falta de materiales en los centros: pizarras, } \\
\text { Tablets, etc. Yo puedo hacer mil cursos, pero si llego al } \\
\text { aula y no tengo los recursos necesarios no puedo poner } \\
\text { en práctica los conocimientos adquiridos" (ENTRE.47). }\end{array}$ \\
\hline & Calidad de la formación. & $\begin{array}{l}\text { "En el centro no hay una persona con verdaderos } \\
\text { conocimientos informáticos y discapacidad que nos } \\
\text { ayude a solucionar los problemas que nos podamos } \\
\text { encontrar.". (ENTRE.35). }\end{array}$ \\
\hline & Distancia geográfica. & $\begin{array}{l}\text { "En nuestro caso si los cursos son online o a distancia } \\
\text { no tenemos dificultades, pero por la zona en la que } \\
\text { vivimos si nos tenemos que desplazar a los cursos que } \\
\text { se realizan en Pamplona nos resulta más difícil.". } \\
\text { (ENTRE.19). }\end{array}$ \\
\hline & Falta de tiempo. & $\begin{array}{l}\text { "La principal barrera es la falta de tiempo ya no solo para } \\
\text { realizar la formación sino para preparar las sesiones en } \\
\text { las que podamos incorporar los conocimientos } \\
\text { adquiridos" (ENTRE.46). }\end{array}$ \\
\hline $\begin{array}{l}\text { Prioridad en la formación: } \\
\text { preferencia de los } \\
\text { informantes claves en la } \\
\text { formación (Respuesta de } \\
\text { opción múltiple). }\end{array}$ & $\begin{array}{l}\text { a) Conocer materiales } \\
\text { tecnológicos específicos. } \\
\text { b) Conocer diferentes } \\
\text { softwares. } \\
\text { c) Saber aplicar estrategias } \\
\text { didácticas y adaptaciones } \\
\text { curriculares. } \\
\text { d) Localizar sitios web con } \\
\text { recursos educativos. } \\
\text { e) Conseguir adaptar un } \\
\text { equipo informático. } \\
\text { f) Conocer instituciones } \\
\text { relacionadas con la } \\
\text { accesibilidad de los sitios } \\
\text { web. }\end{array}$ & (Igual a las subcategorías). \\
\hline
\end{tabular}

Fuente: elaboración propia 


\section{Instrumentos.}

La técnica de recogida de información ha sido la entrevista semiestructurada. Para su validación se optó por aplicar la estrategia de juicio de expertos, mediante el método Delphi, siendo ésta realizada mediante un documento anexado a la entrevista, con una serie de cuestiones abiertas y que fueron entregadas a ocho especialistas en el área de tecnología educativa procedentes de distintas universidades españolas.

\section{Procedimiento.}

El análisis de contenido que se han seguido en esta investigación consta de las siguientes fases: preanálisis, formación del sistema categorial, codificación y análisis e interpretación, utilizando para ello una herramienta informática que facilita el análisis cualitativo de datos, NVivo 11.

El orden de las categorías y la decisión de tomar las respuestas de los entrevistados como correctas o incorrectas han estado sujetas a criterios de concordancia y fiabilidad. Es decir, el análisis realizado, tanto para el proceso de construcción del sistema de categorías (concordancia entre codificadores), como para el momento en el que los codificadores han hecho uso de ese sistema de categorías (fiabilidad), ha dado como resultado un coeficiente kappas excelente por encima de 0.75 (Fleiss, 1981).

\section{Análisis y resultados.}

Apoyándonos en la base empírica de las percepciones de los informantes, recogemos a continuación la red conceptual en la que se muestra la lista de categorías con sus correspondientes subcategorías, así como los resultados obtenidos tras el análisis.

Entre los principales resultados de este estudio, encontramos que el $23 \%$ de entrevistados definen correctamente el concepto de diversidad funcional y el $77 \%$ de los participantes lo hace de forma incorrecta.

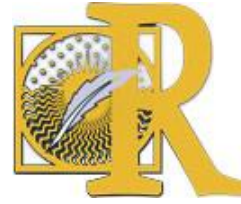

Fecha de recepción: 02-11-2019 Fecha de aceptación: 09-12-2019 
Ilustración 1. Red conceptual, relación entre categorías y subcategorías.

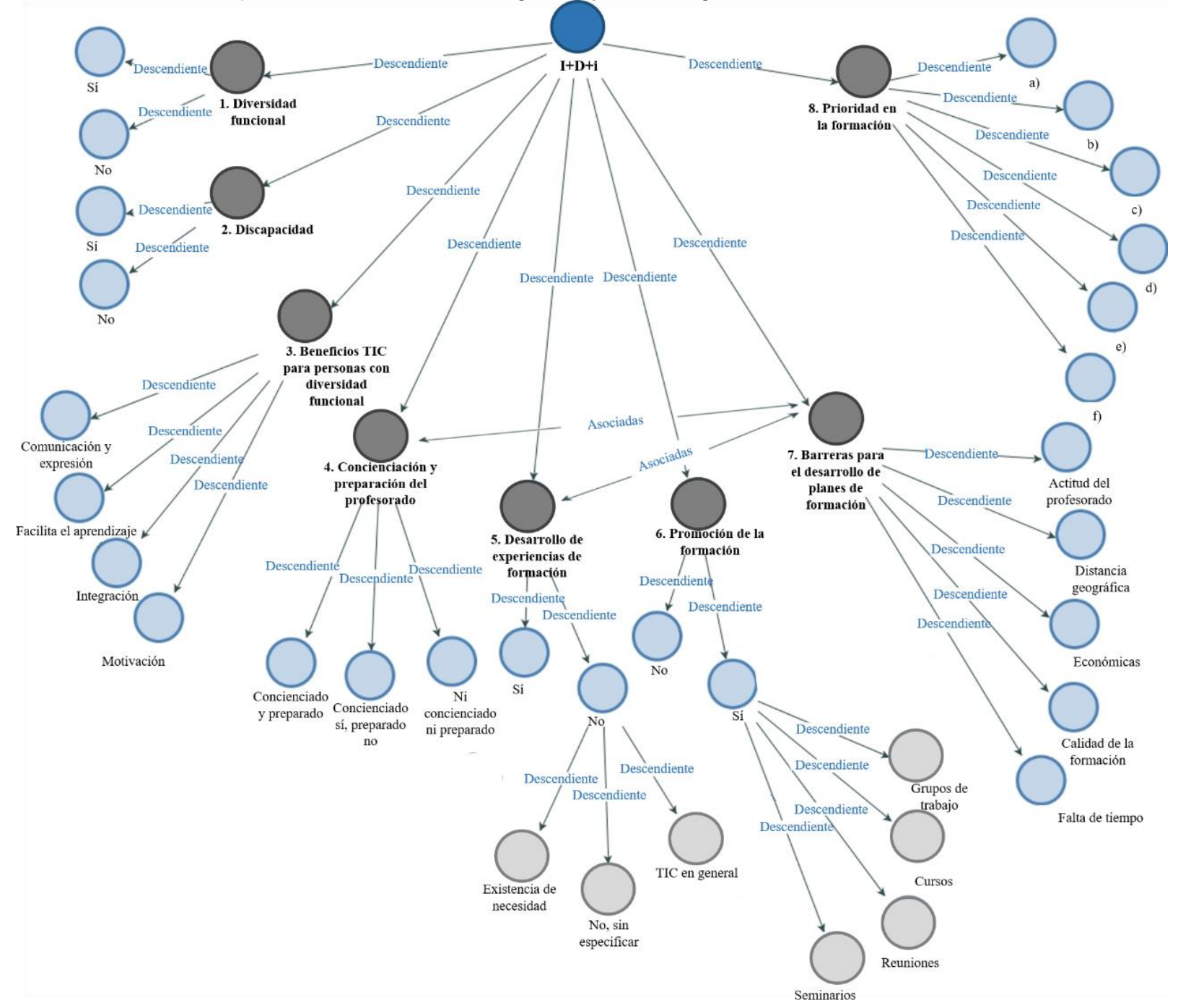

Fuente: elaboración propia.

Respecto a la variable "tipo de centro", ningún informante clave de los centros concertados y privados conoce el significado del término diversidad funcional, sólo en el caso de los entrevistados de los CEP (100\% de las opiniones) sabrían definir dicho término, al igual que el $14 \%$ de los participantes de centros públicos.

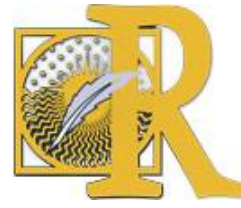

Fecha de recepción: 02-11-2019 Fecha de aceptación: 09-12-2019 
Gráfico 1. Tipo de centro y diversidad funcional.

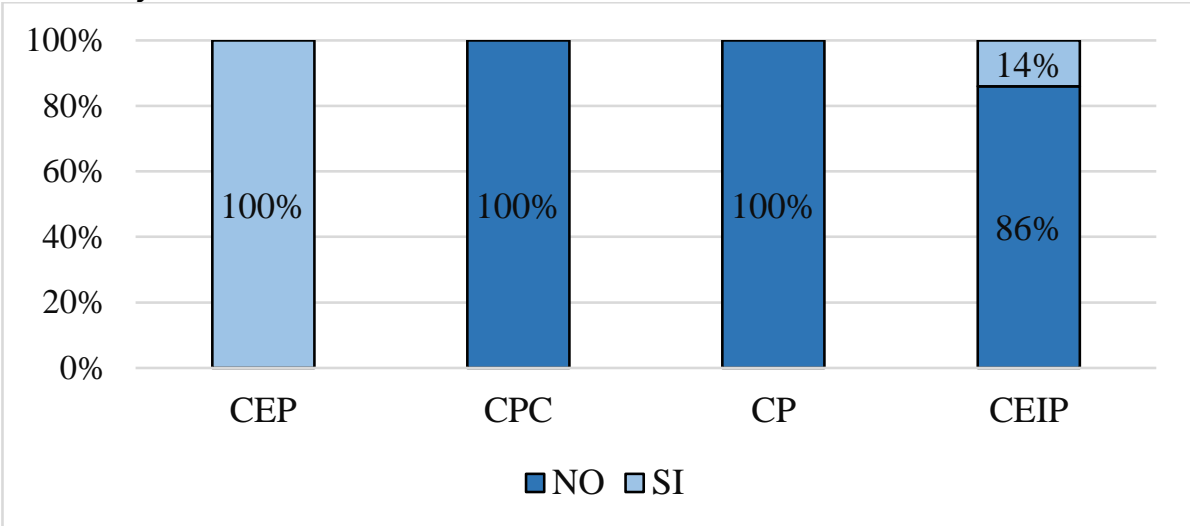

Fuente: elaboración propia.

En cuanto al concepto discapacidad, el porcentaje de respuestas correctas aumentan respecto al obtenido en la definición del término anterior. En este caso, el $85 \%$ de los participantes sabe definir correctamente el término de discapacidad y el $15 \%$ de los entrevistados lo hace de forma incorrecta. El alto porcentaje de respuestas adecuadas se da independientemente del tipo de centro, casi todos los informantes claves de todos los han aportado todas las respuestas correctas en cuanto el concepto discapacidad (100\%). No obstante, un $29 \%$ de los docentes de los CEIP no conocen el concepto de discapacidad.

Del mismo, cabe destacar que los participantes reconocen los beneficios que aportan estas herramientas tecnológicas al alumnado con diversidad funcional (Tabla 3).

Tabla 3. Porcentaje de beneficios que aportan las TIC a las personas con diversidad funcional.

\begin{tabular}{llc} 
& \multicolumn{1}{l}{ SUBCATEGORÍA } & $\%$ \\
\cline { 2 - 3 } Accesibilidad & $43 \%$ \\
\hline $\begin{array}{ll}\text { Comunicación y } \\
\text { expresión }\end{array}$ & $14 \%$ \\
\hline Facilita el aprendizaje & $29 \%$ \\
\cline { 2 - 3 } & Motivación & $14 \%$ \\
\hline
\end{tabular}

Fuente: elaboración propia.

Las subcategorías que han obtenido un mayor porcentaje son: la referida a la "accesibilidad" (43\%) y "facilita el aprendizaje" (29\%), como se pone de manifiesto en expresiones como:

"El principal beneficio que aportan, bajo mi punto de vista, es que permiten acceder, a este tipo de alumnos, a contenidos que antes no podían." (ENTRE.51).

"Pueden ayudar también a compensar en cierta medida algunas limitaciones que estas personas puedan tener" (ENTRE.23).

Teniendo en cuenta los resultados anteriormente mencionados, se consideró relevante preguntar a los entrevistados si creían que el profesorado de Educación Primaria estaba preparado y concienciado para trabajar con alumnado con diversidad funcional. En este

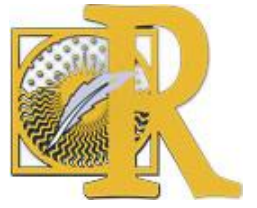

Fecha de recepción: 02-11-2019 Fecha de aceptación: 09-12-2019 
sentido, un $46 \%$ de los informantes claves reconoce que los docentes de Educación Primaria no están ni concienciados ni preparados, frente al 15\% de los entrevistados que opinan que están concienciados y preparados para ello.

Los motivos que afianzan este escaso nivel de concienciación y preparación están relacionados con aspectos como: la edad y/o el desinterés por parte del profesorado, la rápida actualización de herramientas tecnológicas, medios y recursos obsoletos, así como temática relacionada únicamente con profesorado especialista (pedagogía terapéutica o audición y lenguaje).

"No, ni con las Nuevas Tecnologías ni sin las Nuevas Tecnologías. Se tratan de casos muy difíciles y no todo el mundo está preparado para dar una respuesta educativa ajustada a las características de este tipo de alumnos." (ENTRE.01).

"No estamos preparados. Vamos poco a poco, algo de dificultad sí que tenemos, pero con las ganas y el interés nos vamos formando." (ENTRE.43).

Por otra parte, en relación con el tipo de centro educativo, tal y como se muestra en el gráfico 2 , el $100 \%$ de los participantes de centros privados, el $67 \%$ de los de centros concertados y el $44 \%$ de los entrevistados de centros públicos opinan que el profesorado de Primaria no está ni concienciado ni preparado para ayudar al alumnado con diversidad funcional. Sólo el $28 \%$ de los encuestados de los centros públicos consideran que el profesorado de Primaria está concienciado y preparado para ayudar al alumnado con diversidad funcional en el uso de las TIC.

El porcentaje alcanzado por los CEP necesita de una matización. La pregunta realizada a los informantes claves sobre el nivel de concienciación y preparación del profesorado únicamente hacía referencia a los docentes de Educación Primaria. Por tanto, según el 100\% de docentes participantes pertenecientes a CEP, los maestros y maestras de Educación Primaria están concienciados si, preparados no para ayudar al alumnado con diversidad funcional a utilizar las TIC.

Gráfico 2. Porcentaje obtenido sobre el nivel de concienciación y preparación del profesorado según tipo de centro.

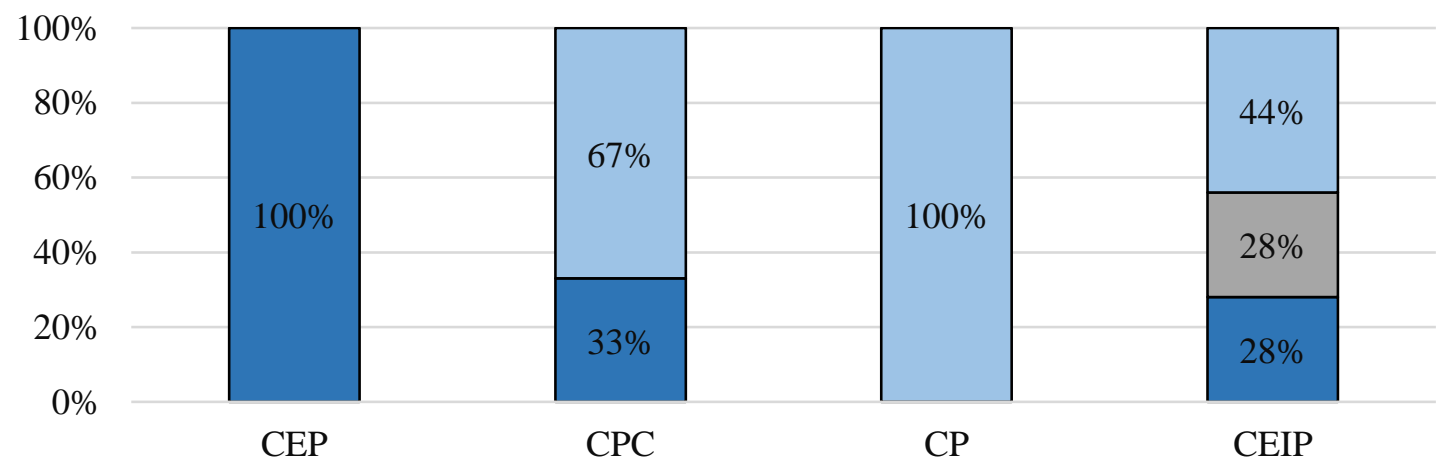

$\square$ Concienciado sí, preparado no $\square$ Concienciado y preparado $\square \mathrm{Ni}$ concienciado, ni preparado

Fuente: elaboración propia.

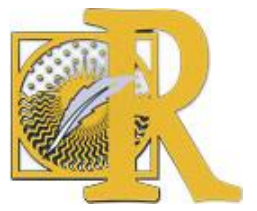

Fecha de recepción: 02-11-2019 Fecha de aceptación: 09-12-2019 
Tras conocer el nivel de concienciación y preparación que posee el profesorado de Educación Primaria en activo, se considera fundamental comprobar el grado de desarrollo de experiencias de formación sobre TIC aplicadas al alumnado con diversidad funcional según la opinión de los entrevistados.

En este sentido, el $77 \%$ de los informantes claves de la Comunidad de Navarra reconoce que la formación es escasa o se centra en el uso de las TIC en general, sin tener en cuenta al alumnado con diversidad funcional, frente al $23 \%$ restante que considera lo contrario.

"En los centros yo creo que no, pero en los centros de profesores yo creo que si existen este tipo de cursos. De todas formas, se puede solicitar formación a asociaciones como la ONCE." (ENTRE.59).

"No, aquí en Navarra por lo menos no. Lo que hacemos es desarrollar cursos TIC para todo el alumnado en general." (ENTRE.10).

De igual modo, consideramos necesario conocer cuáles son los centros que menos realizan actividades de formación sobre TIC aplicadas a personas con diversidad funcional. En este sentido, hemos podido comprobar que el $100 \%$ de los entrevistados de los CEIP y CP considera que no se realizan experiencias de formación sobre TIC aplicadas a personas con diversidad funcional. En cambio, para el $67 \%$ de los participantes de los CPC y el $50 \%$ de los encuestados de los CEP, consideran que si se realiza este tipo de formación.

Ahora bien, partiendo del contexto anterior resulta pertinente conocer si los centros facilitan la realización de experiencias de formación y de qué forma se promociona la formación. El alto porcentaje obtenido (77\%) pone de manifiesto que los centros educativos y los centros de formación promocionan y tratan de ofrecer, a través de diversos medios, actividades de formación para el profesorado de Educación Primaria.

A raíz de los resultados obtenidos se denota una tendencia hacia la realización de cursos, destacando aquellos que tratan las TIC en general y otros que especifican el uso de las TIC con alumnado con diversidad funcional. De igual forma, resaltamos la realización grupos de trabajos, seminarios y/o reuniones.

"En el centro si no hay un alumno con alguna dificultad es muy difícil que se hagan iniciativas de este tipo. Los tenemos un poquito olvidados. Aquí en Navarra se ofrecen muchos cursos relacionados con las TIC y la discapacidad, pero insisto el profesorado solo los realiza si tiene en su aula algún alumno con estas características." (ENTRE.31).

Por otro lado, teniendo en cuenta el tipo de centro hay que resaltar que, en todos los casos, los informantes claves opinan mayoritariamente que son los cursos la actividad más desarrollada, menos el caso de los centros privados donde el $100 \%$ de los entrevistados opinan que no se promociona ningún tipo de formación. Conviene destacar que los centros de formación del profesorado destacan con un valor equiparable a la promoción de los cursos, el desarrollo de grupos de trabajo (gráfico 3).

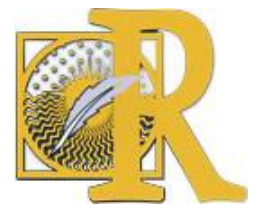

Fecha de recepción: 02-11-2019 Fecha de aceptación: 09-12-2019 
Gráfico 3. Porcentaje obtenido sobre la promoción de la formación según tipo de centro.

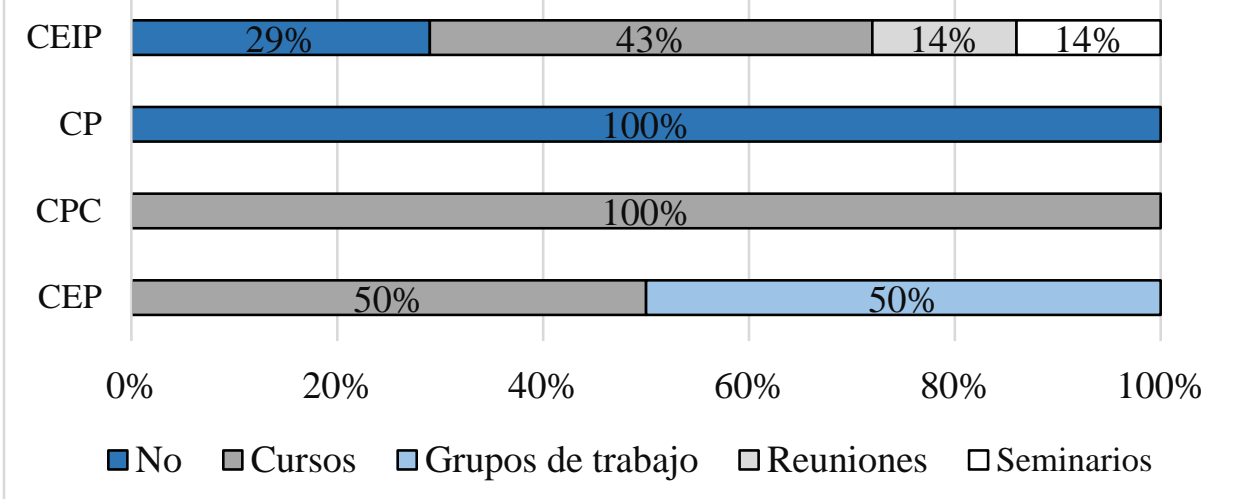

Fuente: elaboración propia.

Una vez comprobado que los centros de la Comunidad de Navarra aseguran que se oferta y promociona la formación $(77 \%)$, pero que no se realizan experiencias de formación suficientes $(77 \%)$ se hace necesario conocer cuáles son las barreras o impedimentos que dificultan el desarrollo de esas actividades.

Tabla 5. Porcentaje de barreras que dificultan la realización de actividades de formación.

\begin{tabular}{lc}
\hline SUBCATEGORÍA & $\%$ \\
\hline Actitud del profesorado & $13 \%$ \\
\hline Calidad de la formación & $7 \%$ \\
\hline Distancia geográfica & $13 \%$ \\
\hline Económicas & $27 \%$ \\
\hline Falta de tiempo & $40 \%$ \\
\hline
\end{tabular}

Fuente: elaboración propia.

Entre las barreras que más dificultan la formación (gráfico 4), se destaca la "falta de tiempo" $(40 \%)$ y los factores "económicos" $(27 \%)$. En esta línea se hacen manifestaciones como:

"La disponibilidad de tiempo, el profesorado se encuentra muy sobrecargado de trabajo: burocracia, preparación de clases, corrección de cuadernos, etc." (ENTRE.21).

"Las principales barreras son la falta de recursos materiales específicos, se solicitan y suelen tardar en proporcionarlos. Supongo que por la gran demanda que tienen y por la falta de presupuestos." (ENTRE.50).

Además, interesa subrayar que teniendo en cuenta la variable "tipo de centro", los informantes claves de los centros de titularidad pública y concertada las principales barreras son las nombradas anteriormente. No obstante, los participantes de centros privados exclusivamente consideran la falta de tiempo, con el $100 \%$ de las respuestas obtenidas. Mientras que, en el caso de los CEP, con un $50 \%$ de las respuestas se considera la barrera actitud del profesorado igual de importante que las económicas.

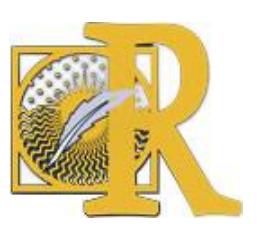

Fecha de recepción: 02-11-2019 Fecha de aceptación: 09-12-2019 
Gráfico 4. Porcentaje de barreras para el desarrollo de planes de formación según el tipo de centro

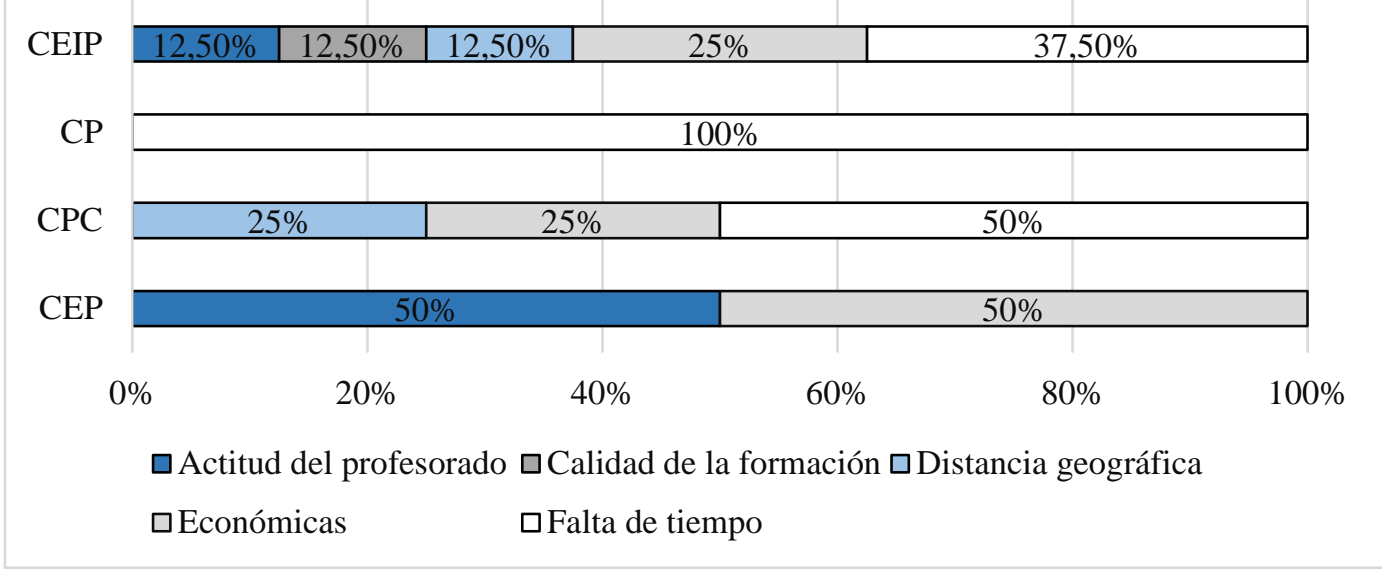

Fuente: elaboración propia

A continuación, con el fin de concretar los aspectos que deben contemplar las actividades de formación dirigidas a maestros de Educación Primaria en cuanto a las TIC aplicadas a personas con diversidad funcional, se muestran varias afirmaciones prioritarias en la formación del profesorado según los participantes.

Entre los resultados más relevantes, los informantes claves consideran prioritarias que las actividades de formación faciliten la labor de "saber aplicar estrategias didácticas y adaptaciones curriculares apoyadas en TIC" (31\%) y "conocer materiales tecnológicos específicos" (18\%).

Finalmente, nos gustaría añadir que, en función del "tipo de centro", los resultados varían considerablemente ya que las opciones más elegidas son la $b$ y $c$. No obstante, en el caso de los centros privados, los entrevistados se decantan de forma equiparable a las opciones referidas por localizar sitios web con recursos educativos, con el $33 \%$ de índice de respuestas. También destaca esta opción $d$, el 19\% de los participantes de los CEIP, coincidiendo el mismo porcentaje de respuestas que refieren con la opción conocer materiales tecnológicos específicos. Por su parte los centros de formación del profesorado valoran también con un $33 \%$ de las respuestas la opción a, equiparándolo a la opción $c$.

\section{Discusión y conclusiones.}

Teniendo en cuenta los resultados obtenidos en el apartado anterior, así como las preguntas de investigación, es posible concluir el estudio con aspectos interesantes como:

RQ1 ¿Se encuentra el profesorado de Educación Primaria de la Comunidad de Navarra, suficientemente preparado y concienciado para el uso y aplicación de las TIC en alumnado con diversidad funcional?

Los informantes claves en esta investigación perciben un bajo nivel de concienciación y conocimiento del profesorado de Educación Primaria respecto a la aplicación de las TIC

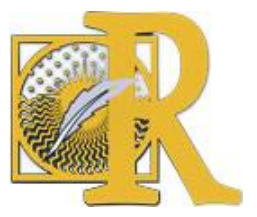

Fecha de recepción: 02-11-2019 Fecha de aceptación: 09-12-2019 
para personas con diversidad funcional. Hallazgos coincidentes con los trabajos de Tello \& Cascales (2015); Morales \& Llorente Cejudo (2016); Cabero, Fernández-Batanero \& Barroso (2016) yFernández-Batanero \& Rodríguez (2017). A pesar de ello, la falta de formación no ha impedido que los entrevistados reconozcan la cantidad de beneficios que aportan las TIC al alumnado con diversidad funcional. Estas evidencias concuerdan con los resultados de otros estudios (Altinay \& Altinay, 2015; Turner-Cmuchal \& Aitken, 2016; Alper \& Goggin, 2017 y Hollier, 2017). Desde esta perspectiva, debemos señalar que la confianza y la educación que recibirá un alumno con diversidad funcional está íntimamente ligada con la formación que posea el docente respecto a ello. Si el fin principal de un sistema educativo de calidad es que se favorezca la inclusión de todos los estudiantes, es esencial que los docentes estén capacitados y conozcan nuevas metodologías que tengan en cuenta el uso de herramientas tecnológicas (Roig, Ferrández, Rodríguez-Cano \& Crespo, 2012; Tello \& Cascales, 2015).

RQ2 ¿Condiciona el tipo de centro docente el nivel de competencia del profesorado para usar las TIC en alumnado con diversidad funcional?

En la Comunidad de Navarra la variable "tipo de centro" sí condiciona la formación del profesorado. En este sentido, destacan los centros de titularidad privada y públicacomo los que menos experiencias de formación realizan y los que menos iniciativa muestran hacia este tipo de formación.

RQ3 ¿Qué factores se asocian a la promoción y el desarrollo de experiencias de formación sobre usos de las TIC en alumnado con diversidad funcional?

La escasa realización de planes de formación no condiciona la promoción de experiencias formativas. De hecho, los participantes reconocen que se oferta a través de diferentes vías, como son la realización de cursos, proyectos o planes de trabajo, seminarios, grupos de trabajo y reuniones en las que se informa al profesorado de todas las actividades que se publican, en las que divulga información o se comparten conocimientos relacionados con el tema.

Del mismo modo, se ha comprobado que las principales barreras que obstaculizan la realización de actividades de formación de TIC y diversidad funcional en la Comunidad de Navarra vienen determinadas por la "falta de tiempo", la "actitud del profesorado" y por factores "económicos". Hallazgo que concuerda con los obtenidos por Ramírez, Cañedo \& Clemente (2011); González \& De Pablos (2015); Villalba, González-Rivera \& Díaz-Pulido (2017) y Fernández-Batanero \& Rodríguez (2017).

Asimismo, en lo referente a la prioridad en la formación se considera necesario que las actividades formativas faciliten la labor de aplicar estrategias didácticas y adaptaciones curriculares apoyadas en TIC, conocer diferentes softwares, así como, aquella formación que proporcione conocimientos sobre materiales tecnológicos específicos para personas con diversidad funcional. En definitiva, se pone de manifiesto la necesidad de una actividad formativa que incluya todos los elementos (materiales, software, aplicación de estrategias y adaptaciones, sitios web, adaptación de equipos informáticos e instituciones accesibles).

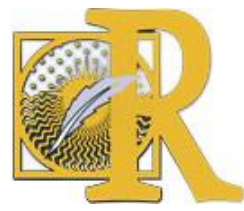

Fecha de recepción: 02-11-2019 Fecha de aceptación: 09-12-2019 
En definitiva, las TIC desempeñan un papel de una enorme riqueza y los profesionales de la educación no pueden permanecer al margen. Asimismo, la interacción entre las TIC y la diversidad funcional por discapacidad, es decir, las formas en las que promuevan la formación e innovación educativa orientada a la equidad, deben suponer un factor educativo de primer orden y una línea prioritaria de investigación (Fernández-Batanero, 2017; FernándezBatanero et al. 2018).

\section{Apoyo.}

Este artículo es parte del resultado de una investigación realizada con el apoyo del Ministerio de Economía y Competitividad Español, en el marco del Plan Estatal de Fomento de la Investigación Científica y Técnica de Excelencia 2013-2016 del Proyecto de investigación denominado: Diagnóstico y Formación del Profesorado para la Incorporación de las TIC en Alumnado con Diversidad Funcional (DIFOTICYD) (EDU2016 75232-P).

\section{Referencias}

- Alper, M. y Goggin, G. (2017). Digital technology and rights in the lives of children with disabilities. New Media \& Society.. Recuperado de: http://journals.sagepub.com/doi/full/10.1177/1461444816686323

- Altinay A. y Altinay, Z. (2015). Examination on ICT integration into Special Education Schools for Developing Countries. TOJET: The Turkish Online Journal of Educational Technology, 14 (3), 70-72.

- Álvarez, J. F., y Gisbert, M. (2015). Grado de alfabetización informacional del profesorado de Secundaria en España: Creencias y autopercepciones. Comunicar, XXIII, 45,187-194, doi: 10.3916/C45-2015-20

- Barrantes, C. G., Casas G. L., y Luengo G. R. (2011). Obstáculos percibidos para la integración de las TIC por los profesores de infantil y primaria en Navarra. Pixel-Bit. Revista de Medios y Educación, 39, 83-94.Cabero, J., Fernández Batanero, J. M. y Barroso, J. (2016). Los alumnos del grado de magisterio: TIC y discapacidad. REDIE. Revista Electrónica de Investigación Educativa, 18(3), 106-120. Recuperado de http://redie.uabc.mx/redie/article/view/965

- Fernández Batanero, J. M. y Bermejo, B. (2012). Actitudes docentes hacia las TIC en centros de buenas prácticas educativas con orientación inclusiva. Enseñanza \& Teaching, 30(1), 45-46.

- Fernández-Batanero, J. M. (2017). IC y diversidad funcional. Hacia la calidad y equidad En López-Meneses, E.; Cobos-Sanchiz, D.; Martín Padilla, A. H.; Molina-García, L. y Jaén Martínez, A. (eds.) (2017). INNOVAGOGÍA 2016. III Congreso Internacional sobre Innovación Pedagógica y Praxis Educativa. Libro de Actas. 28, 29 y 30 de noviembre de 2016. AFOE Formación: Sevilla.

- Fernández-Batanero, J.M. y Rodríguez, A. (2017). TIC y diversidad funcional: conocimiento del profesorado. EJIHPE. European Journal of Investigation in Health, Psychology and Education, 7 (3), 157-175.

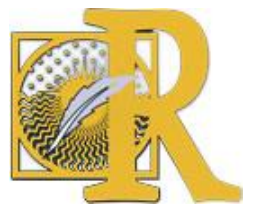

Fecha de recepción: 02-11-2019 Fecha de aceptación: 09-12-2019 
- Fernández-Batanero, J.M., López-Meneses, E., Vázquez-Cano, E. y Moreno-Martínez, N. Má. (2018). Diversidad funcional y tecnologías de la información y la comunicación. Barcelona: Octaedro.

- Fernández Batanero, J. M., Cabero, J. y López, E. (2019). Knowledge and Degree of Training of Primary Education Teachers in Relation to ICT Taught to Students With Disabilities. British journal of educational technology. 50(4), 1961-1978. https://doi.org/10.1111/bjet.12675

- Fernández Batanero, J. M. (2020). TIC y discapacidad: investigación e innovación educativa. Barcelona: Octaedro.

- Fleiss, J. L. (1981). Statistical methods for rates and proportions. New York: John Wiley and Sons.

- González, A. y De Pablos, J. (2015). Factores que dificultan la integración de las TIC en las aulas. Revista de Investigación Educativa, 33 (2), 401-417. doi: http://dx.doi.org/10.6018/rie.33.2.198161

- Hollier, S. (2017). Technology, education and access: A 'fair go' for people with disabilities. 14th International Web for All Conference, W4A.

- Homero, G., Tejedor, F. J. y Calvo, M. I. (2017). Meta-análisis sobre el efecto del software educativo en alumnos con necesidades educativas especiales. Revista de Investigación Educativa, 35 (1), 35-52.

- Morales, P. T. y Llorente Cejudo, M. C. (2016). Formación inicial del profesorado en el uso de Tecnologías de la Información y la Comunicación (TIC) para la educación del discapacitado. Digital Education Review, 30, 123-134.

- OMS. (2001). International Classification of Functioning, Disability and Health. Ginebra, Organización Mundial de la Salud.

- Ortíz, A. M., Almanzán, L., Peñaherrera, M. y Cachón, J. (2014). Formación en TIC de futuros maestros desde el análisis de la práctica en la Universidad de Jaén. Pixel-Bit. Revista de Medios y Educación, 44, 127-142.

- Ramírez, E., Cañedo, I. y Clemente, M. (2011). Las actitudes y creencias de los profesores de secundaria sobre el uso del Internet en sus clases. Comunicar, XIX (38), 47-155.

- Rangel, P. y Peñalosa, E. (2013). Alfabetización digital en docentes de educación superior: construcción y prueba empírica de un instrumento de evaluación. Pixel-Bit. Revista de Medios y Educación, 43, 9-23.

- Roig, R.; Ferrández, S.; Rodríguez-Cano, C. y Crespo, M. (2012). El uso de las TIC en el aula de Educación Especial: percepción de los maestros, en Juan Navarro, María Teresa Fernández, Francisco Javier Soto y Francisco Tortosa (coords.). Respuestas flexibles en contextos educativos diversos. Murcia: Consejería de Educación, Formación y Empleo. Recuperado de: http://diversidad. murciaeduca.es/publicaciones/dea2012/docs/ rroig.pdf

- Romañach, J. y Lobato, M. (2005). Diversidad funcional, nuevo término para la lucha por la dignidad en la diversidad del ser humano, Foro de Vida Independiente. Recuperado de: http://forovidaindependiente.org/wp-content/uploads/diversidad funcional.pdf 
- Rosario, H. y Vásquez, L. (2012). Formación del docente universitario en el uso de tic. Caso de las universidades públicas y privadas. (U. de Carabobo y U. Metropolitana). PixelBit. Revista de Medios y Educación, 41, 163-171.

- Shin, W. S. (2015). Teachers' use of technology and its influencing factors in Korean elementary schools. Technology, Pedagogy and Education, 24, 461-476. doi:10.1080/1475939x.2014.915229.

- Tello, I y Cascales, A. (2015). Las TIC y las necesidades específicas de apoyo educativo: análisis de las competencias tic en los docentes. RIED, 18 (2), 355-383.

- Turner-Cmuchal, M. y Aitken, S. (2016). ICT as a tool for supporting inclusive learning opportunities. Perspectivas Internacionales sobre la Educación Inclusiva, 8, 159-180.

- Villalba, A., González-Rivera, M. D. y Díaz-Pulido, B. (2017). Obstacles perceived by physical education teachers to integrating ICT. Turkish Online Journal of Educational Technology, 16 (1), 83-92. 